\title{
A Novel Compact Rectangular Microstrip Patch Antenna with a Superstrate Element for 2.4GHz WLAN Applications
}

\author{
Goker Sener \\ Department of Electrical-Electronics Engineering, Cankaya University, Ankara, Turkey
}

\begin{abstract}
This paper presents a new compact rectangular microstrip patch antenna with a superstrate element. This antenna operates at $2.4 \mathrm{GHz} \mathrm{TM}_{01}$ fundamental mode, which is suitable for WLAN applications. The patch area is reduced by $50 \%$ by placing three rectangular slots on the ground plane. In order to compensate for the decreased gain due to the size reduction, a high permittivity superstrate is used with $4 \mathrm{~mm}$ thickness and $5 \mathrm{~mm}$ height from the antenna surface. The proposed antenna offers the advantage of occupying half the area of the non-modified rectangular patch while it possesses the same broadside gain of $6-7 \mathrm{~dB}$. The trade-off is the additional antenna height due to the placement of the superstrate element.
\end{abstract}

Key words: Microstrip antennas, Compact antennas, WLAN applications.

\section{Introduction}

Microstrip patch antennas are created by placing metal geometries (patches) on the upper surface of a dielectric structure (substrate) whose bottom surface is grounded. These metal patches are usually in rectangular or circular geometry, and they are available at every frequency between 100 $\mathrm{MHz}$ and $100 \mathrm{GHz}$. General advantages of microstrip antennas are their light weight, low cost, low cross-section profile and compatibility with planar structures. The general drawbacks are their narrow bandwidth and moderate gain. Overall, they have 1-2\% impedance bandwidth and the highest gain of about $6 \mathrm{~dB}$; Therefore, they are used more often in aviation and mobile applications [1].

Microstrip patch antennas are frequently used in 2.4-2.5 GHz ISM (Industrial, Scientific and Medical) applications. For example, in [2], the bandwidth and front-back gain ratio of a microstrip patch antenna are increased by using electromagnetic band gap (EBG) structures within the substrate layer for WLAN applications at $2.4 \mathrm{GHz}$. Also in [3], a compact microstrip patch antenna is developed for WLAN (2.4 GHz), WiMAX $(2.3 \mathrm{GHz}, 2.5 \mathrm{GHz}, 3.5 \mathrm{GHz})$ and Wi-Fi $(2.4 \mathrm{GHz})$ applications.

Miniaturization of patch antennas have important advantages. Today's wireless communication systems require small antennas in mobile phones and similar units. In addition, because array antennas can contain tens or even hundreds of patches, the dimensions of each patch significantly affect the size of the overall antenna. The following methods are generally used to reduce the size of the microstrip patch antennas [4]: First, the permittivity of the dielectric layer can be increased. However, this bounds the electric field tightly within the dielectric substrate, thereby shortening the area that radiates from the edges. Second, the patch can be shorted to ground from one of its

*Corresponding author: Address: Faculty of Engineering, Department of Civil Engineering Sakarya University, 54187, Sakarya TURKEY. E-mail address: caglar@sakarya.edu.tr, Phone: +902642955752 
edges. This technique also reduces the patch dimension; however, the antenna radiates only from one edge, yielding poor radiation efficiency. The third technique is to open meandering slots on the patch surface, or on the ground plane. In this technique, the antenna operates at the same frequency with smaller dimensions, because the surface currents travel further distance due to meandering lines. The disadvantage of this method is that the gain of the antenna decreases as a result of the radiation from the slots [4]. An example of this technique is the microstrip patch in [5] operated at $2.4 \mathrm{GHz}$ having 3 slots on the ground plane of the antenna, establishing the antenna miniaturization by $50 \%$.

One of the methods to compensate for the gain loss in compact patch antennas is to use a di-electric layer (superstrate) at a certain height above the surface of the antenna [1,6]. This di-electric layer acts as a reflector lens that collects the radiation in the direction perpendicular to the antenna surface and increases the gain. Therefore, as the electric permittivity and thickness of this upper layer increases, the gain of the antenna increases proportionally. In literature, there are many examples of superstrate usage for compact patch antennas. For example, in [7], ground shorted compact rectangular patch is designed to operate at $1.84 \mathrm{GHz}$ having a superstrate on top of its substrate with electric permittivity constant $\epsilon_{\mathrm{r}}=79$ and thickness $\mathrm{h}=3.05 \mathrm{~mm}$. The antenna gain of $10.8 \mathrm{~dB}$ is attained. Again, in [8], An FR4 di-electric superstrate is placed on top of a compact micro-strip antenna resulting in a gain of $15.8 \mathrm{~dB}$ at $5.8 \mathrm{GHz}$.

The patch antenna presented in this article is formed by modifying a rectangular microstrip antenna (RMSA) by opening meandering slots on the ground plane. Furthermore, a superstrate with high electric permittivity is used to compensate for the gain loss. The proposed compact patch antenna is compared with a plain rectangular patch antenna using HFSS computer simulation. The results show that the proposed antenna has $50 \%$ less patch surface than the standard RMSA while retaining the broadside gain of $7.24 \mathrm{~dB}$, which is slightly greater than the plain RMSA gain of $6.67 \mathrm{~dB}$. The impedance bandwidth results are $7.5 \%$ versus $1 \%$ for the proposed and the plain RMSA respectively. Thus, both the gain and the bandwidth are improved for the proposed design despite its reduced size. On the other hand, the trade-off is the increased antenna height by $9 \mathrm{~mm}$.

\section{The Compact RMSA with a Superstrate Element}

Based on the technique called the "Cavity Model", the resonant frequency of a plain RMSA at $\mathrm{TM}_{01}$ fundamental mode can be obtained from the following formula $[1,6,9]$

$$
f=2.4 G H z=\frac{1}{2 L \sqrt{\mu \epsilon}}
$$

where $\mathrm{L}$ is the patch length to be determined, and $\mu$ and $\epsilon$ are the electrical properties of the substrate. Solving (1) for $\mu=\mu_{0}$ and $\epsilon=\epsilon_{r} \epsilon_{0}=2.1 \epsilon_{0}, \mathrm{~L}=40 \mathrm{~mm}$ is obtained; however, in reality, this value is slightly larger because of the fringing fields that exist along the edges of the patch. If we take the height of the antenna as $\mathrm{h}=1.6 \mathrm{~mm}$, then the width $(\mathrm{W})$ of the patch can be taken as $\mathrm{W}=30 \mathrm{~mm}$ as it must satisfy the criteria $L>W>h[4]$. 
Table 1. Design parameters and their values in mm's.

\begin{tabular}{lllllllllllll}
\hline $\mathrm{dH}^{*}$ & $\epsilon_{\text {Sub }}{ }^{*}$ & $\epsilon_{\text {Sup }}{ }^{*}$ & patchX & patchY & subX & subY & $\mathrm{dX}$ & $\mathrm{dY}$ & $\mathrm{dsX}$ & $\mathrm{dsY}^{*}$ & $\mathrm{dsH}$ & $\mathrm{dsZ}$ \\
\hline 1.6 & 2.1 & 12 & 40.43 & 30 & 100 & 60 & 20 & 30 & 1 & 26.2 & 5 & 4 \\
\hline
\end{tabular}

$\epsilon_{\text {Sub }}{ }^{*}$ and $\epsilon_{\text {Sup }}{ }^{*}$ are the relative permittivity values of the substrate (RT/duroid 5880) and superstrate layers respectively. $\mathrm{ds}^{*}=26.2 \mathrm{~mm}$ is the total slot length where $1.2 \mathrm{~mm}$ is outside, $25 \mathrm{~mm}$ is inside the patch area. $\mathrm{dH}^{*}$ is the substrate height.

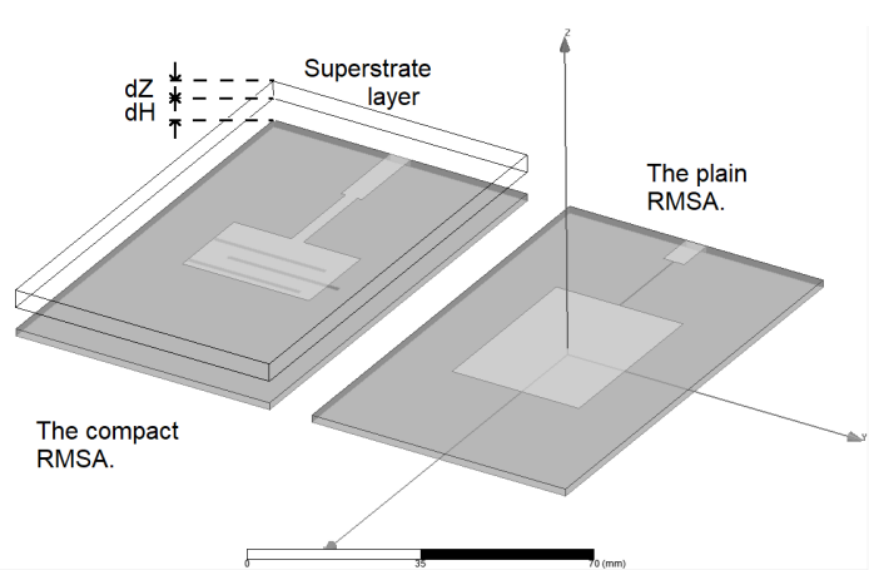

(a)

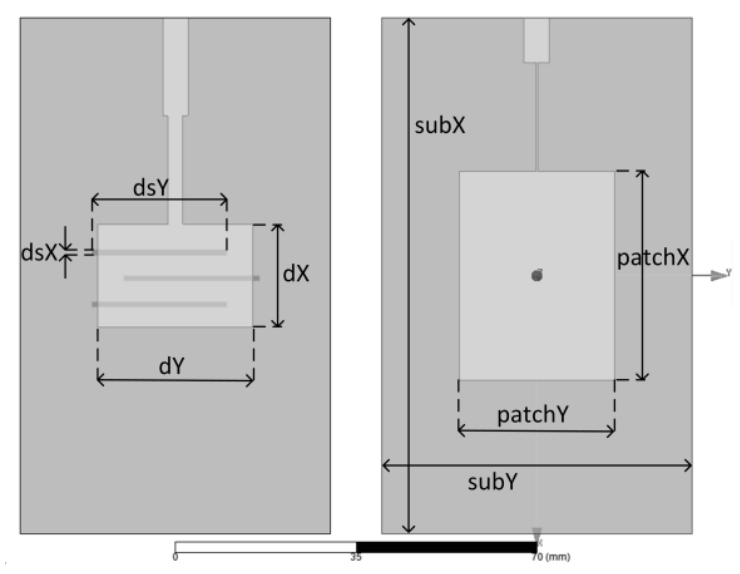

(b)

Figure 1. The proposed compact RMSA and the plain RMSA in (a) 3D view, (b) top view.

The proposed compact antenna and the plain RMSA are shown in Figure 1. The dimensions of the antennas are presented in Table 1. In the proposed design, the patch area is reduced by $50 \%$ by placing 3 meandering slots on the ground plane. The locations and the widths of the slots are determined based on techniques explained in [5]. The length of the slots is adjusted until the desired frequency of $2.4 \mathrm{GHz}$ is attained at half the patch length.

The superstrate layer is made of silicon, and is placed $5 \mathrm{~mm}$ above the antenna surface. It has a thickness of $4 \mathrm{~mm}$, and has the same azimuthal dimensions as the antenna substrate. The antenna is fed by a $50 \Omega$ microstrip transmission line, which is matched to the patch by a quarter wave transformer line.

The impedance bandwidth of the proposed antenna is shown in Figure 2. The graph shows that the plain antenna has an impedance bandwidth of $1 \%$, which is typical for an RMSA. On the other hand, the proposed antenna has an impedance bandwidth of $7.5 \%$, which is a significant improvement, mostly due to the presence of the superstrate.

The principle E-plane $\left(\varphi=0^{0}\right.$ plane or xz-plane) radiation pattern of the antenna is shown in Figure 3. The co-polar (co-pol.) and cross-polar (x-pol.) power gains are obtained as $(7.24 \mathrm{~dB},-45 \mathrm{~dB})$ and $(6.65 \mathrm{~dB},-45 \mathrm{~dB})$ for the proposed RMSA and the plain RMSA respectively. The front-toback ratio is $13.5 \mathrm{~dB}$ for the proposed antenna, and $37 \mathrm{~dB}$ for the plain RMSA. The reason for this reduction is the back radiation from the slots on the ground plane. Placing slots on the front patch would compensate for the front to back ratio loss; however, this would increase the cross-polar radiation, leading to severe radiation impurity. 


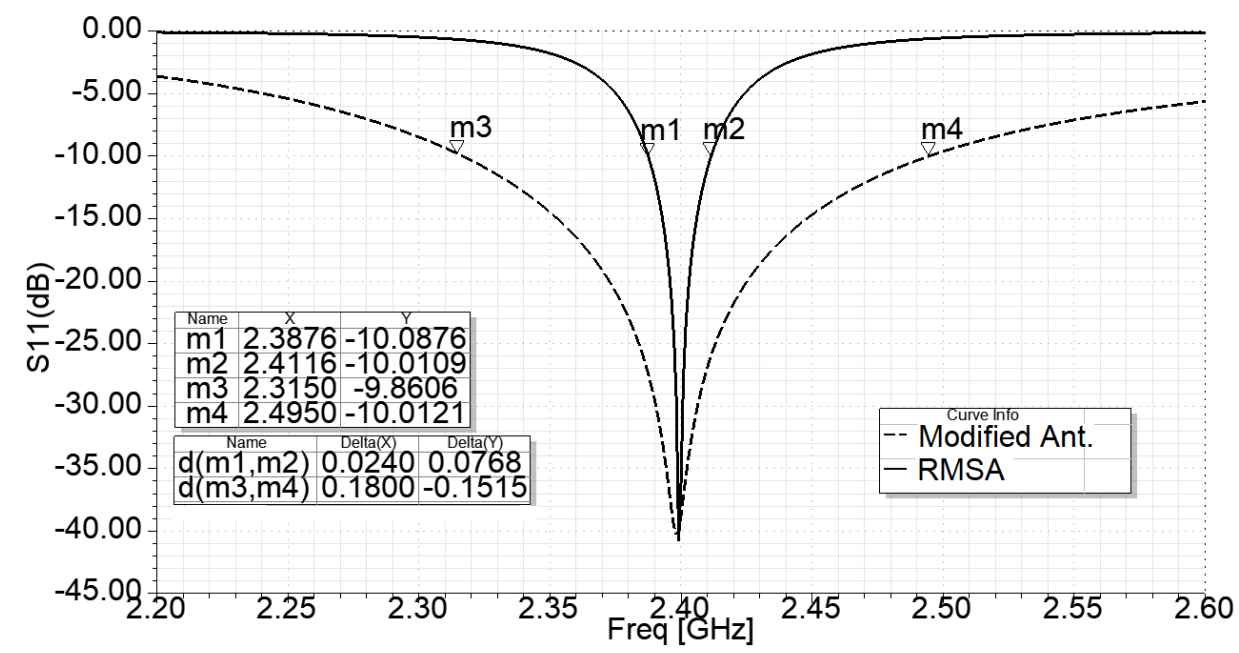

Figure 2. $|\mathrm{S} 11|($ Reflection coefficient) with respect to frequency.

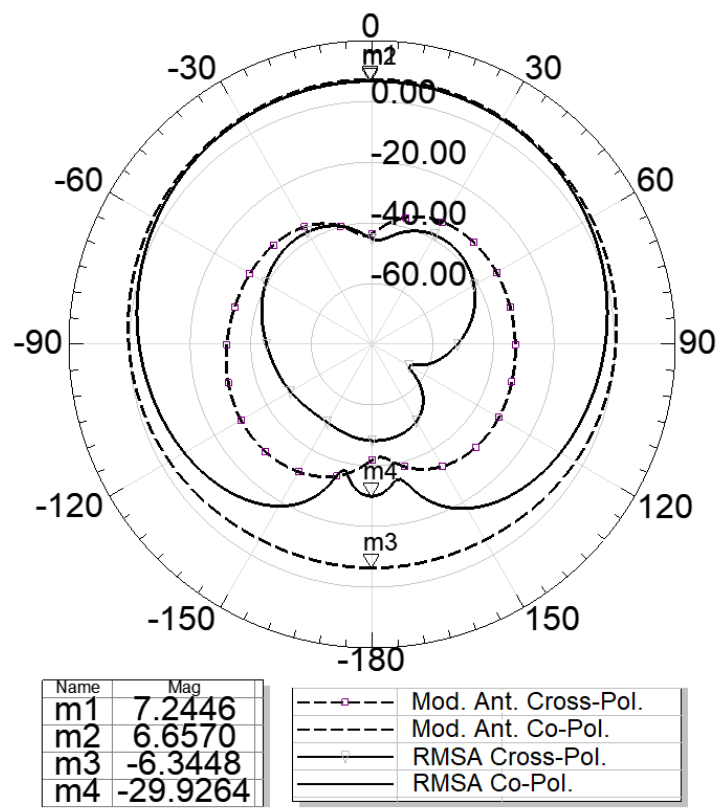

Figure 3. E-Plane radiation pattern for co-pol. $\left(\mathrm{E}_{\theta}\right)$ and $\mathrm{x}-$ pol. $\left(\mathrm{E}_{\varphi}\right)$ electric field components.

\section{Conclusion}

A compact patch antenna is designed to operate at $2.4 \mathrm{GHz}$ ISM band with $50 \%$ less patch area than the plain RMSA. In order to compensate for the gain loss, a silicon superstrate layer is placed $5 \mathrm{~mm}$ above the antenna surface at $4 \mathrm{~mm}$ thickness. Thus, the gain of the antenna is increased to $7.24 \mathrm{~dB}$ for the co-polar field in the broadside direction. The cross polar power gain of the antenna remains the same as the plain RMSA at $-45 \mathrm{~dB}$. The impedance bandwidth of the antenna is increased from $1 \%$ to $7.5 \%$, while the front-rear area intensity ratio is decreased to $13.5 \mathrm{~dB}$ from 
$37 \mathrm{~dB}$ of the plain RMSA. Thus, the proposed antenna is suitable for ISM band applications where high gain, wide bandwidth and small antenna surface are desired.

\section{References}

[1] Garg R, Bhartia P, Bahl I, Ittipiboon A. Microstrip antenna design handbook. Boston, London: Artech House; 2001.

[2] Bhagat P N, Baru V B. Slotted patch antennas with EBG structure for ISM band. 2012 International Conference on Communication, Information \& Computing Technology (ICCICT) Mumbai, India, 2012;1-5.

[3] Yadav A, Verma S. Compact monopole antenna with modified triangular shape for WLAN, WiMAX and Wi-Fi applications. 5th International Conference for Convergence in Technology (I2CT) Pune, India, 2019; 1-5.

[4] Wong K L. Compact and broadband microstrip antennas. New York: John Wiley and Sons; 2002.

[5] Wong K L, Kuo J S, Chiou T W. Compact microstrip antennas with slots loaded in the ground plane. Eleventh International Conference on Antennas and Propagation, 2001; 623-626.

[6] Kumar G, Ray K P. Broadband microstrip antennas. Boston, London: Artech House; 2003.

[7] Huang C Y, Wu J Y. Compact microstrip antenna loaded with very high permittivity superstrate. IEEE Antennas and Propagation Society International Symposium, Atlanta, GA, USA, 1998; 680-683.

[8] Dang D N, Seo C. Compact high gain resonant cavity antenna with via hole feed patch and hybrid parasitic ring superstrate. IEEE Access 2019; 7: 161963-161973.

[9] James J R, Hall P S. Handbook of microstrip antennas. London: Peter Peregrinus Ltd.; 1989. 